\title{
Reverse Engineering Analysis Forensic Malware WEBC2-Div
}

\author{
Raditya Faisal Waliulu*1, Teguh Hidayat Iskandar Alam*2 \\ 12Department of Informatics Engineering, Universitas Muhammadiyah Sorong \\ Sorong, Papua Barat, Indonesia \\ ${ }^{1}$ raditya@um-sorong.ac.id \\ ${ }^{2}$ teguhhidayat@gmail.com \\ accepted on September 24, 2018
}

\begin{abstract}
At this paper focus on Malicious Software also known as Malware APT1 (Advance Persistent Threat) codename WEBC2-DIV the most variants malware has criteria consists of Virus, Worm, Trojan, Adware, Spyware, Backdoor either Rootkit. Although, malware could avoidance scanning antivirus but reverse engineering could be know how dangerous malware infect computer client. Lately, malware attack as a form espionage (cyberwar) one of the most topic on security internet, because of has massive impact. Forensic malware becomes indicator successful user to realized about malware infect. This research about reverse engineering. A few steps there are scanning, suspected packet in network and analysis of malware behavior and disassembler body malware.
\end{abstract}

Keywords: forensic malware, analysis, advance persistent threat, cyberwar, disassemble, static analysis, dynamic analysis

\section{INTRODUCTION}

$\mathbf{R}$ ecently a number of program created for criminal and illegal purpose growth fast. This Program is malware that creates a growing organization, a criminal computer. Definitely, criminal malware take over client's computer and steal personal data, confidential or information of a beneficial nature. this case pressure investigation digital forensic and research security to secure malware attack analysis and use tools that can be relied on beside antivirus.

Today, malware forensics take a part [1]. The aim malware forensic that can identified and analyzed malware which undefined. Many malware created has capable to avoid detection antivirus. Because of that, needs to know analyze malware should be detail about malware capability it self until known impact damage and theft personal data.

Privacy safety, integrity and availability in a real computer system is a challenging task. Increasing amount of system and complex malware between both of them makes secure protection and accurate every system could take time and prone to error.

A discussion of the fundamental challenges and issues/characteristics of malware has been done. Identification of security and privacy issues within this framework are highlighted . Study of the widely used encryption techniques by malware damage in securing sensitive information on cloud is debated. Scope has been set for academicians and researchers. Diverse versions of the encryption techniques surveyed and analyzed to identify harmful or damage for cloud security [2] 


\section{LITERATURE REVIEW}

Malware analysis must be detailed and it take a long time. Malware avoid faced antivirus categorized good one. But, any aspect malware hide from antivirus and it's hard to detected. A few malware forensic tools can show value hidden malware is. In addition, forensic techniques on various tools and plugins more than avoidance analysis techniques. This has become one of the bases for software investigated [3]. Malware analysis one of security computer analyzed malware, learn how and malware's behave. Malware analysis has two method static analysis and dynamic analysis. Analysis static is method disassemble malware without running. But, dynamic analysis is running malware and look for behave itself [4].

Framework or pattern recognition techniques are applied for detection of packed malware binaries. The proposed divided in two phases, first phase it classified packed and non-packed executables. Once an executable is classified as packed, the second phase of classification finds packed benign or packed malware executable. Result framework gain more than $99.9 \%$ accuracy in the first phase of classification and $95 \%$ accuracy in the second phase of classification [5]. High demand Internet data transfer needs is highly dependent on social factors. because the development of technology is increasingly encouraged to understand the mobility of end user needs. not limited only that Human Resources must also be encouraged to know more about the latest technology updates [6]. At this paper focus on malware forensic, a few malware has typical one of virus, trojan, adware, spyware, backdoor, and rootkit capable attack fast to infect operating system [7].

\section{TAXONOMY OF MALICIOUS SOFTWARE}

Created malicious software high growth for cyberwar and spionage there are computer virus which might be confused, such as backdoor, worm and etc [5]. According that following paragraphs offer definitions of these types of malicious software and explanations:

\section{A. Malware}

Contraction of malicious software. Put simply, malware is any piece of software that was written with the intent of doing harm to data, devices or to people. Theses family of malware, including worms, viruses, Trojan horses, backdoors, bombs and rootkits.

B. A Trojan horse

a program that appears to be legal and executed by victim that gives the attacker unauthorized remote access to a system it can be harmful or advantages by attacker.

C. A virus

Recursive code it can replicates itself. In other words, virus could attach in processes or be harm on computer.

D. A worm

Infect computer needs skill social engineering, does not host or human to propagate. Worm works on filetransport or information-transport features on the system, allowing it to travel unaided.

\section{E. Rootkits}

Special tools used to attacker that allows someone to takeover a computer without the computer user/owner knowing about.

F. A backdoor

After take over computer victim's by bypass defense system operating. in could gain unauthorized access and remote. 


\section{ANALYSIS}

At this section we describe our proposed malware analysis schema research forensic malware host and guest Windows XP SP3. Physic host IP 192.168.56.1 and Guest IP 192.168.56.101 this we use bridge interface, fig 1

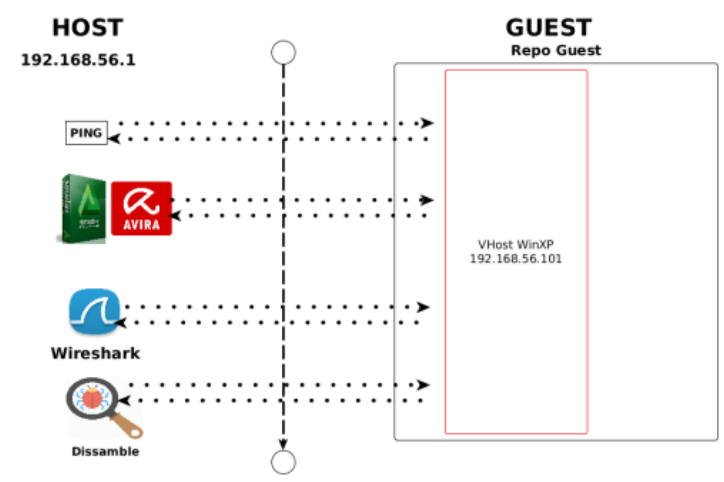

Fig. 1. Proposed model forensic malware WEBC2-DIV

Analysis malware, there are two main techniques for analysis malware that are the most commonly used method was static analysis and dynamic analysis. Static analysis is a method of analysis of malware that done without running the malware, so analysis using this method is much more secure than using the method of dynamic analysis. fig 2

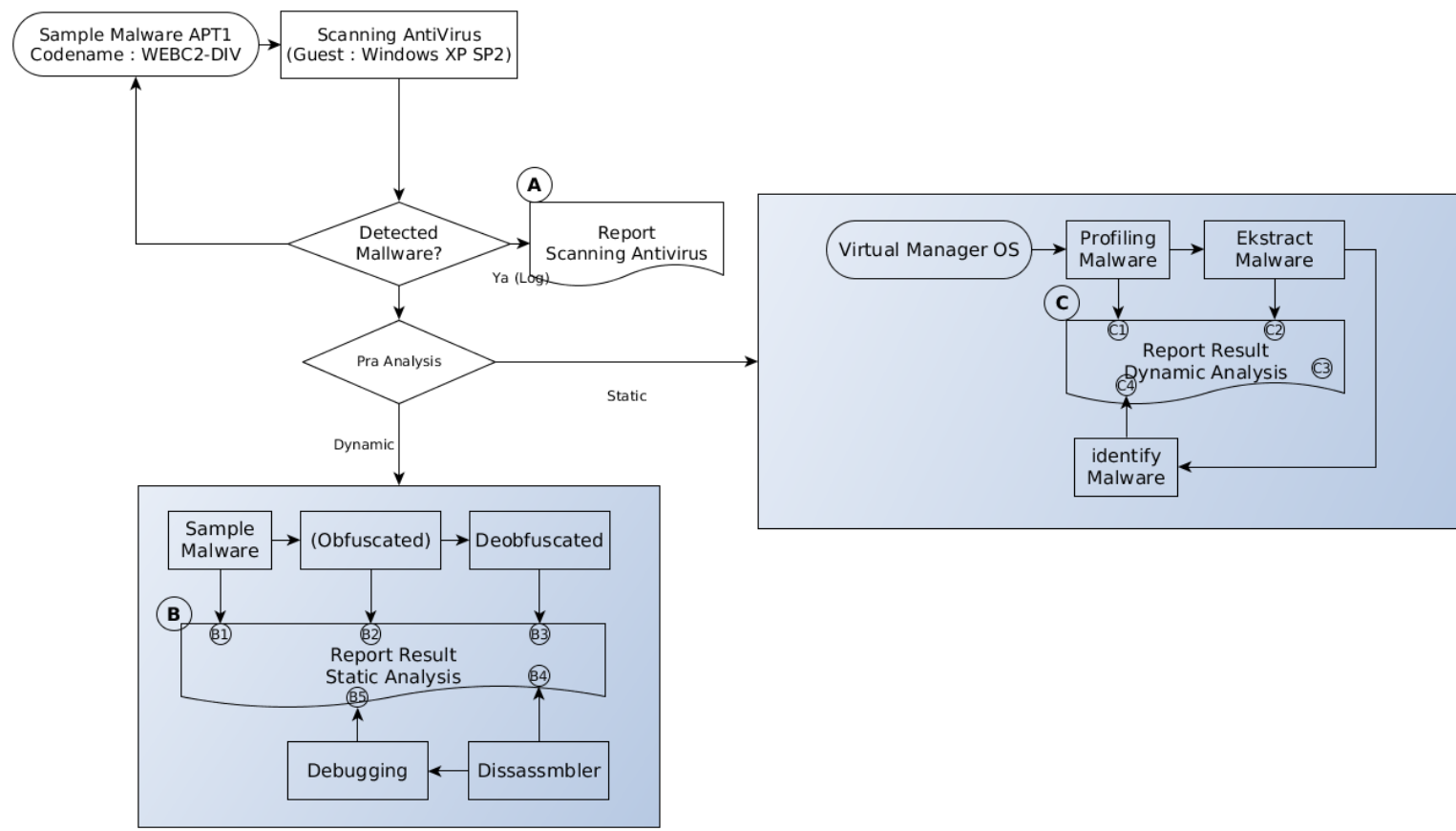

Fig. 2. Works analysis forensic malware WEBC2-DIV

Malware WEBC2-DIV running at Guest, at Figure line blue malware running in name Div.exe, fig 3 


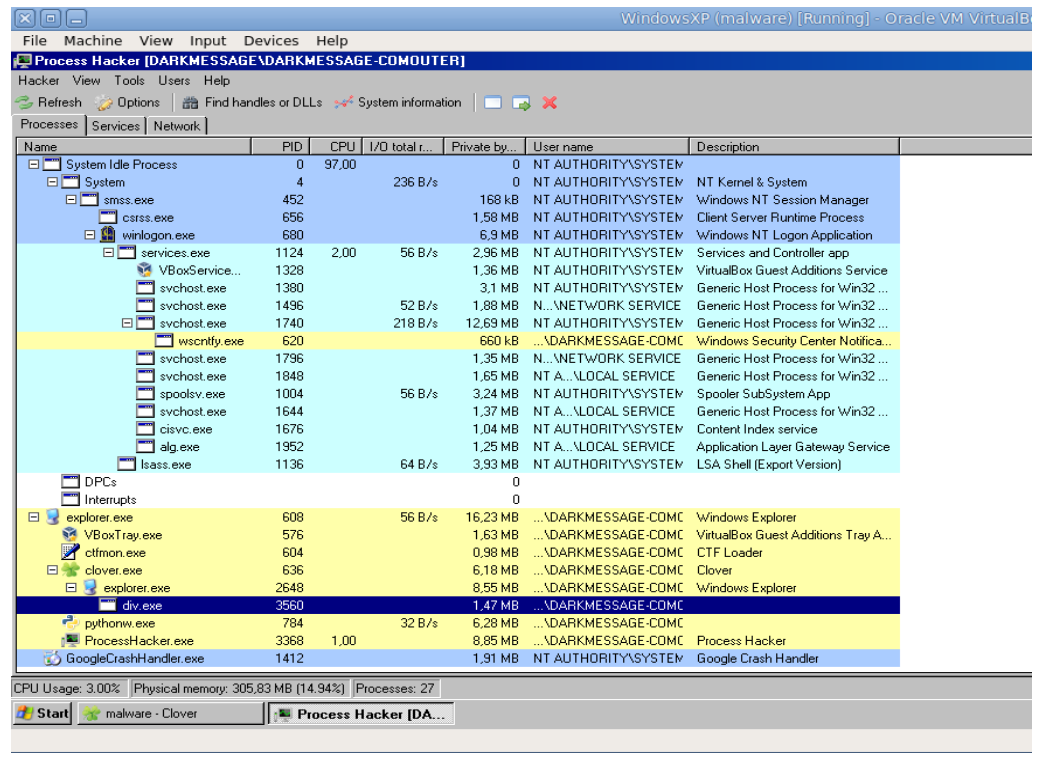

Fig. 3. Process hacker programs to determine WEBC2-DIV

After div.exe running at guest, wireshark on host trying to suspect through network, string cleartext we get and malware trying to connect to thecrowngolf.org, fig 4 .

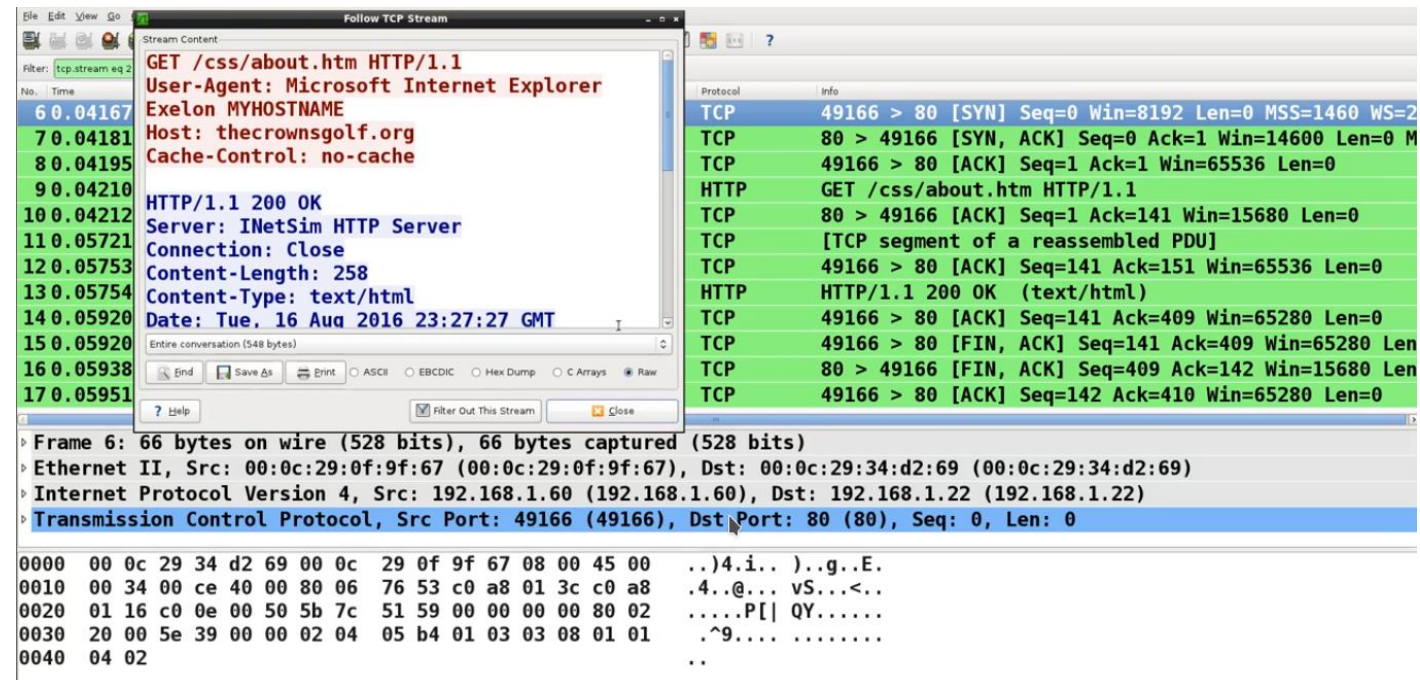

Fig. 4. Host Excute Wireshark

Step before doing reverse engineering, host to do dissembler at OS Parrot OS and Kernel 3.16-04, fig 5.

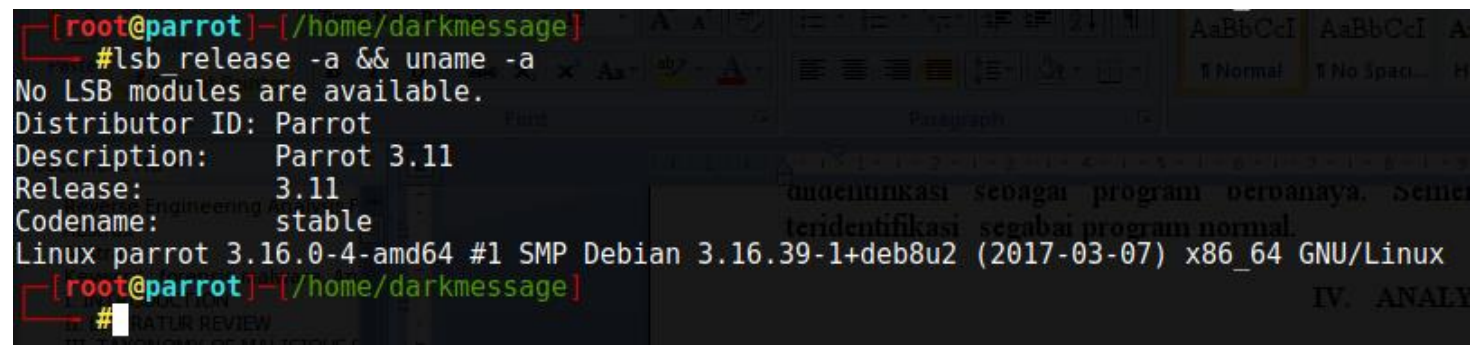

Fig. 5. Host disassembler 
First of all overview about file div.exe malware using Cutter software. Running cutter and insert div.exe we get information about Hash and Library, at fig 6

\section{OVERVIEW}

\section{Info}

$\begin{array}{llll}\text { File: } & \text { arkmessage/Pictures/div.exe } & \text { FD: } & 3 \\ \text { Format: } & \text { pe } & \text { Base addr: } & 0 \\ \text { Bits: } & 32 & \text { Virtual addr: } & \text { True } \\ \text { Class: } & \text { PE32 } & \text { Canary: } & \text { False } \\ \text { Mode: } & -r-x & \text { Crypto: } & \text { False } \\ \text { Size: } & 7168 & \text { NX bit: } & \text { False } \\ \text { Type: } & \text { EXEC (Executable file) } & \text { PIC: } & \text { False } \\ \text { Language: } & & \text { Static: } & \text { False } \\ & & \text { Relro: } & \end{array}$

\section{Hashes}

MD5: 1e5ec6c06e4f6bb958dcbb9fc636009d

SHA1: ed47563dd5cc300716a9ba7946424d538f095ce6

\section{Libraries}

$\begin{array}{ll}\text { Architecture: } & \text { x86 } \\ \text { Machine: } & \text { i386 } \\ \text { OS: } & \text { windows } \\ \text { Subsystem: } & \text { Windows GUI } \\ \text { Stripped: } & \text { True } \\ \text { Relocs: } & \text { True } \\ \text { Endianness: } & \text { little } \\ \text { Compiled: } & \text { on Mar 28 14:35:35 2011 }\end{array}$

\section{wininet.dll}

mfc42.dll

Fig. 6. Overview body malware WEBC2-DIV

It's time to do dissamble on the malware body so it is found that the behavior of the parent malware on the guest and do the planting value in regedit windows address

HKEY_CURRENT_USER/Software/Microsoft/Windows/CurrentVersion/Run. Can be seen in color blocks like fig 7

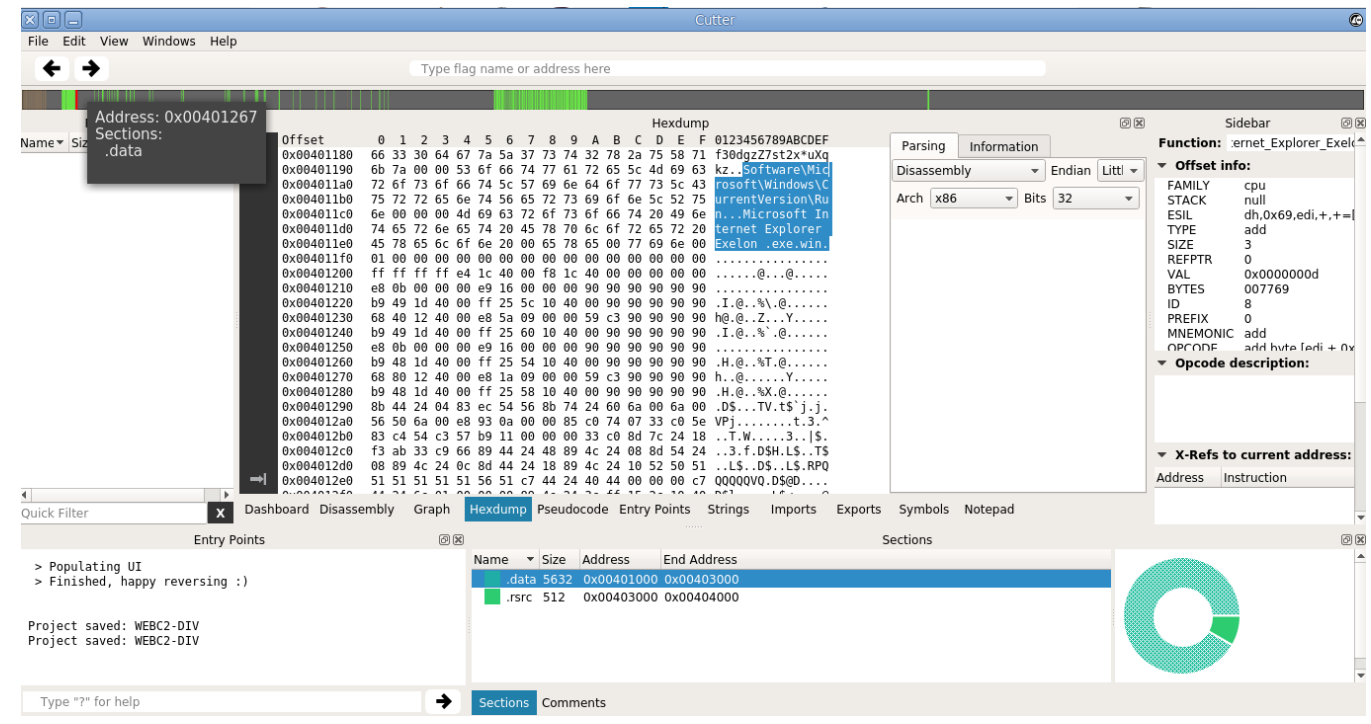

Fig. 7. Address malware on registry Windows

State disassemble not stop at this, body malware got encryption that held important message can infected security computer then could be harmful. Like the encryption block color as fig 8 and fig 9 


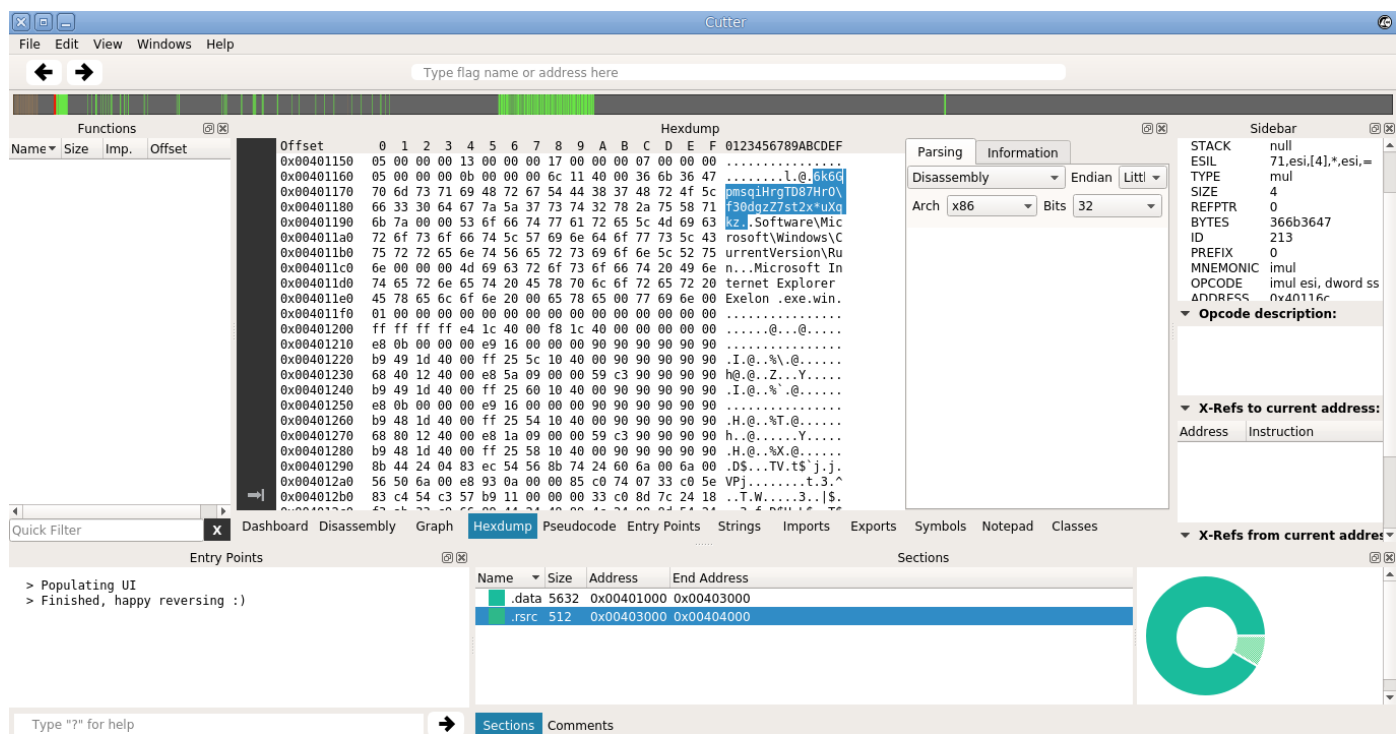

Fig. 8. HexDump value encrypt malware WEBC2-DIV

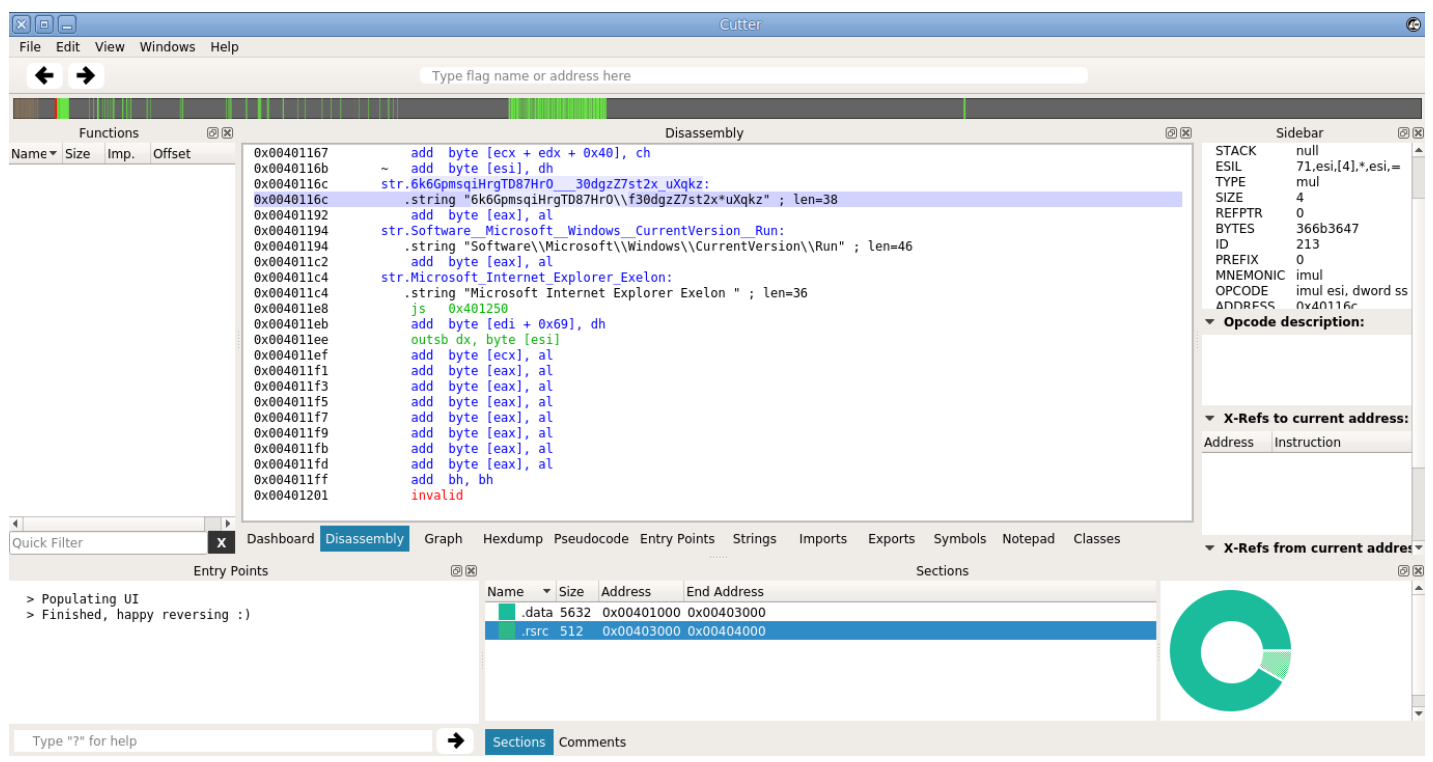

Fig. 9. Value string WEBC2-DIV encrypt on disassembler

Decode encrypt to get information about malware's behave. One another hand, details where's malware sent data to his creator. Result decode we get as fig 10.

\begin{tabular}{|l|l|}
\hline File Edit View Search Terminal Help \\
Decrypt Malware APT1 WEBC2-DIV : http://thecrownsgolf.org/css/about.htm \\
\hline (program exited with code: 0 )
\end{tabular}

Fig. 10. Value string WEBC2-DIV encrypt on disassembler 


\section{Conclusion}

WEBC2-DIV is malicious software best malware to espionage activities performed there are : (1) Phising email, (2) Phising login credential, (3) Backdoor, (4) Remote trojan. This malware well-known since 2010. That does not out posibility malware WEBC2-DIV do update itself by creator then encryption in body malware more difficult than before.

At future research faced automation scanned challenge. Recognize malware by hash then clustering by type malware. This will be innovation and largest contribution to malware research.

[1] PE., dan Grance, T Mell, "The NIST definition of cloud," U.S, 2011.

[2] D dan Nandi, S Devi, "Detection of Packed Malware," in Proceeding SecurIT '12 Proceedings of the First International Conference on Security of Internet of Things, NY, 2012, pp. 22 - 26.

[3] Joshua.I.J., Alan.H., Chen-Ching. L dan Pavel. G Ahmed.F.S., "Towards Automated Malware Behavioral Analysis and Profiling for Digital Forensic Investigation Purposes," in 4th International Conference on Digital Forensics and Cyber Crime ICDF2C 2012, Lafayette, Indiana, USA, 2012.

[4] $\mathrm{H}$ and Lee Jeong K, "Code graph for malware detection, in:Information Networking," ICOIN (International Conference), pp. 1-5, 2008

[5] E. Al., Jebril, I. H., dan Zaqaibeh, B Daoud, "Vol 1. No.2 Computer Virus Stategies and Detection Methods," in Int. J. Open Probles Compt. Math., 2 September 2008.

[6] M., Yegneswaran, V., Saidi, H., Porras, P dan Lee, W Sharif, Eureka: A Framework for Enabling Static Malware Analysis. Berlin, Heidelberg: Springer, 2008, pp. 481-500.

[7] C., Merwe, A.V.D dan Paula, k Mariana, "Secure Computing Benefits, Risk and Controls," IEEE-Information Security, p. Soutch Africa, 2011. 